\title{
A first-order statistical smoothing approximation for the coherent wave field in random porous media
}

\author{
Tobias M. Müller ${ }^{\text {a) }}$ and Boris Gurevich ${ }^{\text {b) }}$ \\ Department of Exploration Geophysics, Curtin University ${ }^{c)}$ GPO Box U1987, Perth, \\ Western Australia 6845, Australia
}

(Received 6 October 2004; revised 24 November 2004; accepted 26 January 2005)

\begin{abstract}
An important dissipation mechanism for waves in randomly inhomogeneous poroelastic media is the effect of wave-induced fluid flow. In the framework of Biot's theory of poroelasticity, this mechanism can be understood as scattering from fast into slow compressional waves. To describe this conversion scattering effect in poroelastic random media, the dynamic characteristics of the coherent wavefield using the theory of statistical wave propagation are analyzed. In particular, the method of statistical smoothing is applied to Biot's equations of poroelasticity. Within the accuracy of the first-order statistical smoothing an effective wave number of the coherent field, which accounts for the effect of wave-induced flow, is derived. This wave number is complex and involves an integral over the correlation function of the medium's fluctuations. It is shown that the known one-dimensional (1-D) result can be obtained as a special case of the present 3-D theory. The expression for the effective wave number allows to derive a model for elastic attenuation and dispersion due to wave-induced fluid flow. These wavefield attributes are analyzed in a companion paper. (C) 2005 Acoustical Society of America. [DOI: 10.1121/1.1871754]
\end{abstract}

PACS numbers: 43.20.Jr, 43.20.Gp, 43.20.-f, 43.20.Wd [JJM] Pages: 1796-1805

\section{INTRODUCTION}

Understanding elastic wave attenuation and dispersion in porous fluid-saturated materials is important in such diverse applications as architectural acoustics, soil and rock mechanics, and exploration seismology. One major cause of elastic wave attenuation is viscous dissipation due to the flow of the pore fluid induced by the passing wave. Wave-induced fluid flow occurs as a passing wave creates local pressure gradients within the fluid phase and the resulting fluid flow is accompanied with internal friction until the pore pressure is equilibrated. The fluid flow can take place on various length scales: for example, from compliant fractures into the equant pores (so-called squirt flow ${ }^{1-3}$ ), or between mesoscopic heterogeneities like fluid patches in partially saturated rocks. ${ }^{4-7}$

Theoretical studies of the elastic wave attenuation due to wave-induced flow go back to the 1970s. In such studies wave propagation in an inhomogeneous porous medium is usually analyzed using Biot's equations of poroelasticity with spatially varying coefficients. ${ }^{8}$ The first models of attenuation due to wave-induced flow considered flow caused by a regular assemblage of inhomogeneities of ideal shape such as two concentric spheres or flat slabs. ${ }^{9-11}$ A general theory of wave propagation in heterogeneous porous media using the double-porosity approach was recently developed by Pride and Berryman ${ }^{12}$ and Pride et al. ${ }^{13}$ In the case of patchy saturation, the results of this theory coincide with those obtained earlier by Johnson ${ }^{14}$ using a slightly different method. Although mathematical formulations in the theories Pride and Berryman ${ }^{12}$ and Johnson ${ }^{14}$ allow for arbitrary geometry of the inhomogeneities, closed-form expressions for

\footnotetext{
a)Electronic mail: tobias.muller@geophy.curtin.edu.au

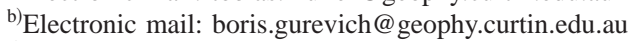

${ }^{c}$ URL: http://www.geophysics.curtin.edu.au
}

attenuation and phase velocity can only be obtained for a regular system of simple geometrical shapes such as concentric spheres.

In real rocks heterogeneities are more likely to be spatially distributed in a random fashion. Therefore, it is desirable, as suggested by Lopatnikov and Gurevich, ${ }^{15}$ to model wave-induced flow using the theory of waves in random media. ${ }^{16}$ For one-dimensional (1-D) porous media, Gurevich and Lopatnikov ${ }^{17}$ showed that elastic wave attenuation in a randomly layered porous medium differs significantly from attenuation in periodically layered porous media. ${ }^{10,18}$ This suggests that the effects of three-dimensional inhomogeneities on elastic wave wave attenuation and dispersion in porous media may also be different for random and periodic spatial configurations. One approach to this problem is to consider a homogeneous porous medium with randomly distributed discrete, regularly shaped 3-D inclusions. However, so far only the attenuation due to conversion scattering at spherical inclusions from fast $P$ waves into Biot's slow $P$ wave is known. ${ }^{19}$

Our purpose in this paper is to analyze the effect of wave-induced fluid flow on the dynamic characteristics of coherent elastic waves propagating in a porous medium whose properties are continuous random functions of position. Our approach is based on the statistical wave theory, as applied to Biot's equations of poroelasticity. ${ }^{8}$ We restrict our analysis to the case of mesoscopic inhomogeneities, that is, inhomogeneities whose characteristic size $a$ is much larger than the typical size of pores or grains $a_{\text {pore }}$ but, at the same time, much smaller than the wavelength of the propagating elastic wave, $\lambda$ :

$$
a_{\text {pore }} \ll a \ll \lambda \text {. }
$$

In other words, we ignore pore-scale heterogeneities, which 




FIG. 1. Sketch of a heterogeneous porous medium where only the bulk modulus of the drained-framework-of-grains fluctuates. The homogeneous background medium is characterized by the bulk modulus $K_{d}^{B}$. There are inhomogeneities with higher bulk moduli, that is $K_{d}^{(1)}>K_{d}^{B}$, and other inhomogeneities with lower bulk modulus, $K_{d}^{(2)}<K_{d}^{B}$. The inhomogeneities have a length scale of $a$ that is much larger than the typical pore scale $a_{\text {pore }}$. During the compression cycle of a wave there will be fluid flow from inhomogeneities with $K_{d}^{(2)}$ into the background and flow from the background into inhomogeneities with $K_{d}^{(1)}$. During the extension cycle of the wave the fluid flow becomes reversed.

allows the application of Biot's equations for a poroelastic continuum. ${ }^{8}$ We model wave propagation in the inhomogeneous porous medium using Biot's equations of poroelasticity with randomly varying coefficients (see Fig. 1). By using a Green's function approach, these partial differential equations transform into a system of integral equations. This latter system is solved by means of the method of statistical smoothing which is widely used in problems of electromagnetic, acoustic, and elastic wave propagation. ${ }^{20-22}$ More precisely, we employ a first-order statistical smoothing approximation that sometimes is referred to as a Bourret approximation. ${ }^{16,23}$ For poroelastic media, this method was earlier employed to compute $P$-wave attenuation and dispersion due to conversion scattering from fast $P$ waves into Biot's slow waves in a randomly layered porous medium. ${ }^{17}$ We follow this strategy and analyze the conversion scattering from fast $P$ waves into Biot's slow wave in 3-D randomly inhomogeneous porous media. Biot's slow wave is a highly dissipative wave mode. Therefore, the use of the first-order statistical smoothing approximation to the conversion scattering problem in Biot's equations of poroelasticity quantifies the dissipation of wave field energy due to energy transfer from the coherent component of the fast $P$ wave into the dissipative slow $P$-wave mode. This is different from the usual application of the method of smoothing to energy conserving wave systems, where an apparent dissipation (socalled scattering attenuation) results from the energy transfer from the coherent component of the wave field into the incoherent component.

In light of previous results for randomly layered porous media, it is useful to introduce some simplifications from the outset. First of all, we restrict our analysis to low frequencies. Specifically, we assume that frequency $\omega$ is much smaller than the critical Biot frequency $\omega_{\mathrm{B}}=\phi \eta / \kappa_{0} \rho_{f}$ :

$$
\omega \ll \omega_{\mathrm{B}},
$$

where $\phi$ and $\kappa_{0}$ denote the porosity and permeability of the composite material, while $\rho_{f}$ and $\eta$ are the density and viscosity of the pore fluid. Condition (2) implies that the standard Biot's viscoinertial attenuation and dispersion (the socalled Biot's global flow mechanism) is neglected and the $P$ wave number $k_{p}$ is real. Furthermore, at low frequencies the slow wave is much slower than the fast $P$ wave and therefore the ratio of $k_{p}$ to slow $P$ wave number $k_{p s}$ is a small parameter:

$$
\frac{\left|k_{p}\right|}{\left|k_{p s}\right|} \ll 1 .
$$

Wherever applicable we make use of relation (3), being aware of the underlying low-frequency assumption.

The strategy of this paper is as follows. First, we apply the method of Green's functions to Biot's equations of poroelasticity in order to represent the wavefield due to point source excitation (Sec. II). Next, we derive an integral wavefield representation for the case when the coefficients in Biot's equations exhibit a randomly fluctuating component. This wavefield representation (also called the scattering equation) is then converted into an integral equation for Green's function for the inhomogeneous medium. In Sec. III we apply a perturbation method to compute an approximation for the mean of the Green's function. From this Green's function we derive and analyze an explicit expression for the effective, complex $P$ wave number that accounts for the conversion scattering from $P$ into slow $P$ waves. The range of applicability of our theory is analyzed in Sec. IV. Finally, in Sec. V we show how the results of the aforementioned 1-D theory can be recovered as a limiting case of the more general 3-D theory. A detailed analysis of attenuation and dispersion as functions of frequency and the potential applicability of these wavefield signatures to interpret the effect of wave-induced flow in real rocks is presented in a companion paper. $^{24}$

\section{FORMULATION OF THE POROELASTIC SCATTERING PROBLEM}

\section{A. Green's function approach for Biot's equations of poroelasticity}

In order to study dynamic effects of elastic wave propagation in porous media, we base our analysis on Biot's equation of poroelasticity. ${ }^{8}$ Using index notation-summation over repeated indices is assumed and partial derivatives are denoted as ${ }_{, i}$ or $\partial_{i}$-we can write the equations of motion in the frequency domain (the time-harmonic dependency $\exp (-i \omega t)$ is omitted $)$,

$$
\begin{aligned}
& \rho \omega^{2} u_{i}+\rho_{f} \omega^{2} w_{i}+\tau_{i j, j}=0, \\
& \rho_{f} \omega^{2} u_{i}+q \omega^{2} w_{i}-p_{, i}=0,
\end{aligned}
$$

where $\tau_{i j}$ is the total stress tensor, $p$ the fluid pressure, while $u_{i}$ and $w_{i}$ are the components of the solid and relative fluid displacement vectors, respectively. The relative fluid displacement is defined as $w_{i}=\phi\left(U_{i}-u_{i}\right)$, where is $U_{i}$ the 
fluid displacement. The densities of the solid and fluid phase are denoted by $\rho_{g}$ and $\rho_{f}$ so that the bulk density is given by $\rho=\phi \rho_{f}+(1-\phi) \rho_{g}$, where $\phi$ is porosity. The parameter $q$ is defined as $q=i \eta /\left(\omega \kappa_{0}\right)$, where $\eta$ is viscosity and $\kappa_{0}$ permeability. We note that this definition of $q$ is a consequence of the low-frequency assumption (2).

In order to obtain a closed system of wave equations in the displacements $u_{i}$ and $w_{i}$, we complement the equations of motion with the stress-strain relations for an isotropic poroelastic medium, ${ }^{8}$

$$
\begin{aligned}
& \tau_{i j}=G\left[u_{i, j}+u_{j, i}-2 \delta_{i j} u_{j, j}\right]+\delta_{i j}\left[H u_{j, j}+C w_{j, j}\right], \\
& p=-C u_{j, j}-M w_{j, j} .
\end{aligned}
$$

Here $G$ is the porous-material shear modulus, and $H$ is the undrained, low-frequency $P$-wave modulus given by Gassmann's equation:

$$
H=P_{d}+\alpha^{2} M,
$$

where

$$
M=\left[(\alpha-\phi) / K_{g}+\phi / K_{f}\right]^{-1} .
$$

In Eqs. (8)-(9), $P_{d}=K_{d}+4 / 3 G$ is the $P$-wave modulus of the drained frame, $\alpha=1-K_{d} / K_{g}$ is the Biot-Willis coefficient, $C=\alpha M$, and $K_{g}, K_{d}$, and $K_{f}$ denote the bulk moduli of the solid phase, the drained frame, and the fluid phase, respectively. Symbol $\delta_{i j}$ is Kronecker's delta (the identity tensor).

It is expedient to write the above system of coupled wave equations in matrix form:

$$
\left[\begin{array}{cc}
L_{i k}^{(1)} & L_{i k}^{(2)} \\
L_{i k}^{(3)} & L_{i k}^{(4)}
\end{array}\right] \cdot\left[\begin{array}{l}
u_{k} \\
w_{k}
\end{array}\right]=\mathbf{0}
$$

where we defined the linear differential operators as follows:

$$
\begin{aligned}
& L_{i k}^{(1)}=\rho \omega^{2} \delta_{i k}+\partial_{j} G\left[\delta_{j k} \partial_{i}+\delta_{i k} \partial_{j}-2 \delta_{i j} \partial_{k}\right]+\partial_{i} H \partial_{k}, \\
& L_{i k}^{(2)}=\rho_{f} \omega^{2} \delta_{i k}+\partial_{i} C \partial_{k}, \\
& L_{i k}^{(3)}=L_{i k}^{(2)}, \\
& L_{i k}^{(4)}=q \omega^{2} \delta_{i k}+\partial_{i} M \partial_{k} .
\end{aligned}
$$

Note that in inhomogeneous media all the poroelastic parameters are functions of position. In the presence of point sources, the right-hand side of Eq. (10) can be written as

$$
-\left[\begin{array}{c}
F_{i}^{0} \delta\left(r_{i}-r_{i}^{\prime}\right) \\
f_{i}^{0} \delta\left(r_{i}-r_{i}^{\prime}\right)
\end{array}\right]
$$

where $F_{k}^{0}$ and $f_{k}^{0}$ represent constant forces applied to the bulk and fluid phase, respectively, and $\delta\left(r_{i}-r_{i}^{\prime}\right)$ denotes the Dirac delta function. The response of system (10) to point sources of the form (15) can be formulated as ${ }^{25}$

$$
\left[\begin{array}{c}
u_{i} \\
w_{i}
\end{array}\right]=\left[\begin{array}{cc}
G_{i k}^{F} & G_{i k}^{f} \\
G_{i k}^{f} & G_{i k}^{w}
\end{array}\right] \cdot\left[\begin{array}{c}
F_{k}^{0} \\
f_{k}^{0}
\end{array}\right],
$$

where $G_{i k}^{F}, G_{i k}^{f}$, and $G_{i k}^{w}$ denote the Green's tensors. Thus, the point source response of system (10) in an isotropic unbounded medium is described by three Green's tensors. Explicit expressions for the $G_{i k}$ 's for a homogeneous medium are given by Pride and Haartsen ${ }^{25}$ and reproduced in Appendix A. The wavefields observed at position $\mathbf{r}$ due to arbitrary point sources $F_{i}$ and $f_{i}$, applied at position $\mathbf{r}^{\prime}$, can be expressed by a convolution equation of the form

$$
\left[\begin{array}{c}
u_{i}(\mathbf{r}) \\
w_{i}(\mathbf{r})
\end{array}\right]=\int_{V^{\prime}} d^{3} \mathbf{r}^{\prime}\left[\begin{array}{cc}
G_{i k}^{F}\left(\mathbf{r}-\mathbf{r}^{\prime}\right) & G_{i k}^{f}\left(\mathbf{r}-\mathbf{r}^{\prime}\right) \\
G_{i k}^{f}\left(\mathbf{r}-\mathbf{r}^{\prime}\right) & G_{i k}^{w}\left(\mathbf{r}-\mathbf{r}^{\prime}\right)
\end{array}\right] \cdot\left[\begin{array}{c}
F_{k}\left(\mathbf{r}^{\prime}\right) \\
f_{k}\left(\mathbf{r}^{\prime}\right)
\end{array}\right] .
$$

On the basis of Eq. (17), we now derive a wavefield representation in a randomly inhomogeneous medium.

\section{B. The basic scattering equation}

In randomly inhomogeneous porous media, all poroelastic parameters can be presented as random fields $X(\mathbf{r})$. To be more specific, we assume that each of these poroelastic parameters is the sum of a constant background value, $\bar{X}$, and a fluctuating part, $\widetilde{X}(\mathbf{r})$, so that

$$
X=\bar{X}+\widetilde{X}=\bar{X}\left(1+\varepsilon_{X}\right),
$$

where $\varepsilon_{X}=\tilde{X} / \bar{X}$ denotes the relative fluctuations. The average over the ensemble of the realizations (denoted by $\langle\cdot\rangle$ ) of $\varepsilon_{X}$ is assumed to be zero: $\left\langle\varepsilon_{X}\right\rangle=0$. The spatial correlation function of two random fields is defined as

$$
B_{X X}(\delta \mathbf{r})=\left\langle\varepsilon_{X}(\mathbf{r}+\delta \mathbf{r}) \varepsilon_{X}(\mathbf{r})\right\rangle,
$$

where the dependence of $B$ on the difference vector $\delta \mathbf{r}$ only is a consequence of the assumption of statistically homogeneous random fields, ${ }^{16}$ which we use throughout this paper. The variance of the random process $\varepsilon_{X}$ will be denoted as $B_{X X}(0)=\left\langle\varepsilon_{X}^{2}\right\rangle=\sigma_{X X}^{2}$. Using (18), the differential operators $L_{i k}$ can be also decomposed as ${ }^{21}$

$$
L_{i k}=\bar{L}_{i k}+\tilde{L}_{i k},
$$

where the perturbing operator $\widetilde{L}_{i k}$ satisfies $\left\langle\widetilde{L}_{i k}\right\rangle=0$. As is typical for statistical wave problems, in the following we assume that the constant part $\bar{X}$ and statistical properties of the fluctuations $\varepsilon_{X}$ are known. Therefore, rather than seeking the solution of Eq. (17) for a given realization $\varepsilon_{X}$, we seek statistical moments of the solution for given statistical properties of fluctuations.

The substitution of (20) into matrix equation (10) yields

$$
\left[\begin{array}{cc}
\bar{L}_{i k}^{(1)} & \bar{L}_{i k}^{(2)} \\
\bar{L}_{i k}^{(2)} & \bar{L}_{i k}^{(4)}
\end{array}\right] \cdot\left[\begin{array}{c}
u_{k} \\
w_{k}
\end{array}\right]=-\left[\begin{array}{cc}
\widetilde{L}_{i k}^{(1)} & \widetilde{L}_{i k}^{(2)} \\
\widetilde{L}_{i k}^{(2)} & \widetilde{L}_{i k}^{(4)}
\end{array}\right] \cdot\left[\begin{array}{c}
u_{k} \\
w_{k}
\end{array}\right],
$$

In the most general case, the perturbing operators $\widetilde{L}_{i k}$ contain fluctuations of all poroelastic moduli and densities. The right-hand side of (21) can be thought of as a source term in the homogeneous system (10) due to the presence of inhomogeneities (so-called secondary sources). Thus, Eq. (21) can be understood as an inhomogeneous equation with constant coefficients, whose formal solution can be written by substituting the source term into Eq. (17):

$$
\left[\begin{array}{c}
u_{i} \\
w_{i}
\end{array}\right]=\left[\begin{array}{c}
u_{i}^{0} \\
w_{i}^{0}
\end{array}\right]+\int_{V} d V\left[\begin{array}{cc}
G_{i j}^{F} & G_{i j}^{f} \\
G_{i j}^{f} & G_{i j}^{w}
\end{array}\right] \cdot\left[\begin{array}{cc}
\widetilde{L}_{j k}^{(1)} & \widetilde{L}_{j k}^{(2)} \\
\widetilde{L}_{j k}^{(2)} & \widetilde{L}_{j k}^{(4)}
\end{array}\right] \cdot\left[\begin{array}{c}
u_{k} \\
w_{k}
\end{array}\right] .
$$


Equation (22) is the basic poroelastic scattering equation. The total wavefields $u_{i}$ and $w_{i}$ are composed of wavefields propagating in the homogeneous background medium, $u_{i}^{0}$ and $w_{i}^{0}$, and scattered wavefields (the second term). By definition, $u_{i}^{0}$ and $w_{i}^{0}$ satisfy the homogeneous equation (10). The scattered wavefields are represented by volume integrals whose kernels involve the Green's tensors and the secondary sources. The scattered wavefields vanish if there are no fluctuations in the medium parameters. The integration volume encompasses the inhomogeneous part of the medium, which in our case is the whole 3-D space. We note that Eq. (22) is closely related to the representation integral of the scattered field in an unbounded medium (for the poroelastic formulation of this theorem we refer to Norris ${ }^{26}$ and Pride and Haartsen ${ }^{25}$ ). According to Eq. (17), the wavefields can be represented as a convolution of Green's tensors with the source function. Let us denote Green's tensors for the homogeneous background medium by ${ }^{0} G_{i k}^{F, f, w}$ and for the inhomogeneous medium by $G_{i k}^{F, f, w}$. Substituting these wavefield representations into Eq. (22), we obtain an equation for the Green's tensors of the inhomogeneous medium,

$$
\begin{aligned}
{\left[\begin{array}{cc}
G_{i m}^{F} & G_{i m}^{f} \\
G_{i m}^{f} & G_{i m}^{w}
\end{array}\right]=} & {\left[\begin{array}{cc}
{ }^{0} G_{i m}^{F} & { }^{0} G_{i m}^{f} \\
{ }^{0} G_{i m}^{f} & { }^{0} G_{i m}^{w}
\end{array}\right]+\int_{V} d V\left[\begin{array}{cc}
{ }^{0} G_{i j}^{F} & { }^{0} G_{i j}^{f} \\
{ }^{0} G_{i j}^{f} & { }^{0} G_{i j}^{w}
\end{array}\right] } \\
& \cdot\left[\begin{array}{cc}
\widetilde{L}_{j k}^{(1)} & \widetilde{L}_{j k}^{(2)} \\
\widetilde{L}_{j k}^{(2)} & \widetilde{L}_{j k}^{(4)}
\end{array}\right] \cdot\left[\begin{array}{cc}
G_{k m}^{F} & G_{k m}^{f} \\
G_{k m}^{f} & G_{k m}^{w}
\end{array}\right] .
\end{aligned}
$$

In order to simplify the equations that follow, we introduce a shorthand notation. The latter equation can be symbolically rewritten as

$$
\mathbf{G}=\mathbf{G}^{0}+\int \mathbf{G}^{0} \widetilde{\mathbf{L}} \mathbf{G}
$$

where $\mathbf{G}, \mathbf{G}^{0}$, and $\widetilde{\mathbf{L}}$ represent matrices, whose elements are tensors of rank two, and matrix multiplication rules apply. In (24) we also omitted the integration volume for brevity.

\section{FIRST-ORDER STATISTICAL SMOOTHING OF BIOT'S EQUATIONS OF POROELASTICITY}

\section{A. Mean Green's tensor}

We will now analyze Eq. (24) using a statistical approach. Since the matrix of perturbing operators $\widetilde{\mathbf{L}}$ in Eq. (24) contains fluctuating medium parameters, the resulting matrix of Green's tensors also contains randomly fluctuating elements. Because individual realizations of the random wavefields are never known, it is natural to analyze the statistical moments of G. Solving Eq. (24) by iteration we obtain the scattering series

$$
\mathbf{G}=\mathbf{G}^{0}+\int \mathbf{G}^{0} \widetilde{\mathbf{L}} \mathbf{G}^{0}+\iint \mathbf{G}^{0} \widetilde{\mathbf{L}} \mathbf{G}^{0} \widetilde{\mathbf{L}} \mathbf{G}^{0}+\iiint \cdots .
$$

Averaging this equation by the ensemble of realizations and regrouping the scattering terms yields

$$
\overline{\mathbf{G}}=\mathbf{G}^{0}+\iint \mathbf{G}^{0} \mathbf{Q} \overline{\mathbf{G}},
$$

where $\overline{\mathbf{G}}=\langle\mathbf{G}\rangle$ is the matrix of mean Green's tensors, and $\mathbf{Q}$ is the matrix operator defined as

$$
\mathbf{Q}=\left[\begin{array}{cc}
Q_{i k}^{(1)} & Q_{i k}^{(2)} \\
Q_{i k}^{(3)} & Q_{i k}^{(4)}
\end{array}\right]=\left\langle\widetilde{\mathbf{L}} \mathbf{G}^{0} \tilde{\mathbf{L}}+\int \tilde{\mathbf{L}} \mathbf{G}^{0} \tilde{\mathbf{L}} \mathbf{G}^{0} \tilde{\mathbf{L}}+\int \ldots\right\rangle .
$$

Operator $\mathbf{Q}$ given by Eq. (27) corresponds to the kernel-ofmass operator in the acoustic formulation. ${ }^{16}$ The linear integral equation in $\overline{\mathbf{G}}$ [Eq. (26)] is the poroelastic analog of the Dyson equation (see also Gurevich and Lopatnikov, ${ }^{27}$ where an analogous equation for the mean field is derived). It is not possible to obtain an exact solution of Eq. (26). A first-order statistical smoothing consists in the first-order truncation of the infinite series expression for the operator $\mathbf{Q}$. Then, we obtain the following approximation for the mean Green's tensor:

$$
\begin{aligned}
\overline{\mathbf{G}} & =\mathbf{G}^{0}+\iint \mathbf{G}^{0}\left\langle\widetilde{\mathbf{L}} \mathbf{G}^{0} \widetilde{\mathbf{L}}\right\rangle \overline{\mathbf{G}} \\
& =\mathbf{G}^{0}+\iint \mathbf{G}^{0} \mathbf{Q}^{B} \overline{\mathbf{G}} .
\end{aligned}
$$

The truncation of the series (27) implies that the first-order statistical smoothing is valid when $\left|\varepsilon_{X}\right| \ll 1$, i.e., when the absolute value of the relative fluctuations of $X$ is a small parameter. Note also that the elements of matrix operator $\mathbf{Q}^{B}$ only contain terms involving the second statistical moment of the fluctuating parts of the $\widetilde{L}_{i k}$ 's, that is, they are of the order $O\left(\varepsilon^{2}\right)$. Higher-order correlations are neglected within the accuracy of the first-order statistical smoothing approximation.

Since Eq. (29) contains a double volume convolution, it is expedient to work with its spatial Fourier transform:

$$
\overline{\mathbf{g}}=\mathbf{g}^{\mathbf{0}}+\left(8 \pi^{3}\right)^{2} \mathbf{g}^{\mathbf{0}} \mathbf{q} \overline{\mathbf{g}}
$$

where $\overline{\mathbf{g}}, \mathbf{g}^{\mathbf{0}}$, and $\mathbf{q}$ denote the spatial Fourier transforms of $\overline{\mathbf{G}}, \mathbf{G}^{\mathbf{0}}$, and $\mathbf{Q}^{B}$, respectively (see Appendix A for a definition of the Fourier transform pair). Equation (30) is of the same form as the equation for the mean Green's function in acoustic random media. However, in contrast to the acoustic case, Eq. (30) is not a simple algebraic equation for the mean Green's tensors, but a system of four tensorial equations for the three unknown mean Green's tensors. Formally, we can express the solution of (30) in the form $\overline{\mathbf{g}}=\mathbf{W}^{-1} \mathbf{g}^{0}$, where $\mathbf{W}^{-\mathbf{1}}$ is the inverse of the matrix $\mathbf{W}=\mathbf{I}-\left(8 \pi^{3}\right)^{2} \mathbf{g}^{\mathbf{0}} \mathbf{q}$ with the identity tensor $\mathbf{I}$. However, the computation of $\mathbf{W}^{-\mathbf{1}}$ is cumbersome. Instead, we are looking for a more feasible way to compute some elements of $\overline{\mathbf{g}}$. Carrying out the necessary matrix multiplications in (30), we find that this system splits up into two pairs of coupled equations. Since we are only interested in the characteristics of the fast $P$ wave, which are exclusively contained in the Green's tensor $\bar{g}^{F}$ [see also Eqs. (16) and (A1)], we analyze only those two equations that involve $\bar{g}_{i k}^{F}$. We obtain

$$
\begin{aligned}
\bar{g}^{F}= & g^{F}+\left(8 \pi^{3}\right)^{2}\left[g^{F} q^{(1)} \bar{g}^{F}+g^{F} q^{(2)} \bar{g}^{f}+g^{f} q^{(3)} \bar{g}^{F}\right. \\
& \left.+g^{f} g^{(4)} \bar{g}^{f}\right],
\end{aligned}
$$




$$
\begin{aligned}
\bar{g}^{f}= & g^{f}+\left(8 \pi^{3}\right)^{2}\left[g^{f} q^{(1)} \bar{g}^{F}+g^{f} q^{(2)} \bar{g}^{f}+g^{w} q^{(3)} \bar{g}^{F}\right. \\
& \left.+g^{w} q^{(4)} \bar{g}^{f}\right],
\end{aligned}
$$

where we omitted subscripts for brevity. The quantities $g$ without an upper bar denote the background space Green's tensors. Since all quantities $q^{(i)}(i=1, \ldots, 4)$ are of the order $O\left(\varepsilon^{2}\right), \bar{g}^{f}$ is also of the order $O\left(\varepsilon^{2}\right)$. Inserting the expression for $\bar{g}^{f}$ [Eq. (32)] into Eq. (31) and neglecting terms of higher order than $O\left(\varepsilon^{2}\right)$, we obtain

$$
\begin{aligned}
\bar{g}^{F}= & g^{F}+\left(8 \pi^{3}\right)^{2}\left[q^{F} q^{(1)} \bar{g}^{F}+g^{F} q^{(2)} g^{f}+g^{f} q^{(3)} \bar{g}^{F}\right. \\
& \left.+g^{f} q^{(4)} g^{f}\right] .
\end{aligned}
$$

Equation (33) is an implicit equation for the mean Green's tensor $\bar{g}^{F}$. Because of its tensorial character, an explicit solution for $\bar{g}^{F}$ is still difficult to construct. Note, however, that we are not interested in the mean Green's tensor itself but only in a mean $P$ wave number contained in $\bar{g}^{F}$.

\section{B. Effective wave number of the fast $\boldsymbol{P}$-wave}

In order to extract an effective wave number from Eq. (33) we have to introduce further simplifications. Because of the assumption of small fluctuations in the medium parameters $(\varepsilon \ll 1)$, we can expect that the fluctuations of the wavefield are also small if the wavelengths are much larger than the size of the inhomogeneities. Then, we can assume that mean Green's tensor $\bar{g}_{i k}^{F}(\mathbf{K})$ is of the same functional form as a background Green's tensor $g_{i k}^{F}(\mathbf{K})$ given by Eq. (A8), however, involving some effective $P$-wave number (and also effective bulk density).

Let us construct a simple case, where most of the Green's tensor components vanish. This can be achieved using the following procedure: We consider an incoming, plane $P$ wave propagating in the $x_{3}$ direction (i.e., only the displacement component $u_{3}$ is nonzero). The resulting coherent $P$ wave in the inhomogeneous medium will also propagate in the $x_{3}$ direction. Therefore, only the tensor components $i$ $=j=3$ of $g_{i j}^{F}$ need to be analyzed. Noting that in this case the Green's tensor $g_{i k}^{F}(\mathbf{K})$ yields the largest contribution for the spatial wave number $K=k_{p}$, we can approximate the full Green's tensor (A8) by

$$
g_{33}^{F} \approx \frac{-1}{8 \pi^{3} \rho \omega^{2}}\left(1+\frac{K^{2}}{k_{p}^{2}-K^{2}}\right) .
$$

We assume that the mean Green's tensor component is given by

$$
\bar{g}_{33}^{F} \approx \frac{-1}{8 \pi^{3} \bar{\rho} \omega^{2}}\left(1+\frac{K^{2}}{\bar{k}_{p}^{2}-K^{2}}\right),
$$

where $\bar{k}_{p}$ is the searched-for effective $P$ wave number. Substituting Eq. (34) for $g^{F}$ and Eq. (35) for $\bar{g}^{F}$ into Eq. (33) we obtain, after algebraic manipulations,

$$
\bar{k}_{p} \approx k_{p}\left(1+\frac{4 \pi^{3}}{\rho \omega^{2}} q_{33}^{(1)}\right) .
$$

Here we neglected terms that contain combinations of the tensor components $q_{33}^{(i)}$. This introduces no additional inac- curacy because higher-order correlations are neglected within the accuracy of the first-order statistical smoothing $O\left(\varepsilon^{2}\right)$.

The remaining problem is the evaluation of $q_{33}^{(1)}$ in (36), or equivalently, of $Q_{33}^{(1)}$ in space domain. In explicit form, from the first term in the expansion of $\mathbf{Q}$ as given by Eq. (27), we obtain

$$
\begin{aligned}
Q_{i k}^{(1)}\left(\mathbf{r}^{\prime}-\mathbf{r}^{\prime \prime}\right)= & \left\langle\widetilde{L}_{i j}^{(1)}\left(\mathbf{r}^{\prime}\right) G_{j l}^{F}\left(\mathbf{r}^{\prime}-\mathbf{r}^{\prime \prime}\right) \widetilde{L}_{l k}^{(1)}\left(\mathbf{r}^{\prime \prime}\right)\right. \\
& +2 \widetilde{L}_{i j}^{(1)}\left(\mathbf{r}^{\prime}\right) G_{j l}^{f}\left(\mathbf{r}^{\prime}-\mathbf{r}^{\prime \prime}\right) \widetilde{L}_{l k}^{(2)}\left(\mathbf{r}^{\prime \prime}\right) \\
& \left.+\widetilde{L}_{i j}^{(2)}\left(\mathbf{r}^{\prime}\right) G_{j l}^{w}\left(\mathbf{r}^{\prime}-\mathbf{r}^{\prime \prime}\right) \widetilde{L}_{l k}^{(2)}\left(\mathbf{r}^{\prime \prime}\right)\right\rangle,
\end{aligned}
$$

where for statistically homogeneous random media both $Q_{i k}$ and $G_{i k}$ depend only on the difference vector $\mathbf{r}^{\prime}-\mathbf{r}^{\prime \prime}$. It is interesting to note that in the elastic limit, only the first term of $Q_{i k}^{(1)}$ is nonzero. In the poroelastic case we have to analyze all three terms. Expression (37) involves the perturbing operators $\widetilde{L}_{i j}^{(1)}$ and $\widetilde{L}_{i j}^{(2)}$ (but not $\widetilde{L}_{i j}^{(4)}$ ). Let us now specify the perturbing operators resulting from (11) and (12),

$$
\begin{aligned}
& \widetilde{L}_{i k}^{(1)}=\widetilde{\rho} \omega^{2} \delta_{i k}+\partial_{j} \widetilde{G}\left[\delta_{j k} \partial_{i}+\delta_{i k} \partial_{j}-2 \delta_{i j} \partial_{k}\right]+\partial_{i} \widetilde{H} \partial_{k}, \\
& \widetilde{L}_{i k}^{(2)}=\widetilde{\rho}_{f} \omega^{2} \delta_{i k}+\partial_{i} \widetilde{C} \partial_{k},
\end{aligned}
$$

for a particular situation in which we can find an explicit analytical expression for the right-hand side of Eq. (37). In the following, we neglect fluctuations of the densities $\rho$ and $\rho_{f}$. This is possible because of the restriction to low frequencies. It can be shown that incorporation of density fluctuations yields a correction to the background $P$ wave number [the second term in Eq. (36)], which scales with $\omega^{3}$, whereas the other fluctuations result in a $\omega^{2}$ dependence, as shown below. This simplification is also in accordance with the 1-D result, ${ }^{17}$ where the density fluctuations do not appear in the final expression for the effective $P$ wave number though these fluctuations were not neglected a priori. These simplifications yield

$$
\begin{aligned}
& \widetilde{L}_{i k}^{(1)}=\partial_{k} \widetilde{G} \partial_{i}+\partial_{j} \delta_{i k} \widetilde{G} \partial_{j}-2 \partial_{i} \widetilde{G} \partial_{k}+\partial_{i} \widetilde{H} \partial_{k}, \\
& \widetilde{L}_{i k}^{(2)}=\partial_{i} \widetilde{C} \partial_{k} .
\end{aligned}
$$

A detailed computation of the three $Q_{i k}^{(1)}$ terms in Eq. (37) using the perturbing operators (40) and (41) is provided in Appendix B. The result in the wave number domain can be represented as

$$
q_{33}^{(1)}=q^{H H}+q^{H G}+q^{H C}+q^{G G}+q^{G C}+q^{C C},
$$

where

$$
\begin{aligned}
q^{H H}= & \frac{1}{8 \pi^{3}} k_{p}^{2}\left(\frac{H^{2}}{P_{d}} B_{H H}(0)\right. \\
& \left.+\frac{C^{2}}{N} k_{p s}^{2} \int_{0}^{\infty} r B_{H H}(r) \exp \left[i k_{p s} r\right] d r\right),
\end{aligned}
$$




$$
\begin{aligned}
q^{H G}= & -\frac{1}{3 \pi^{3}} k_{p}^{2}\left(\frac{G H}{P_{d}} B_{H G}(0)\right. \\
& \left.+\frac{\alpha^{2} M G}{P_{d}} k_{p s}^{2} \int_{0}^{\infty} r B_{P M}(r) \exp \left[i k_{p s} r\right] d r\right) \\
q^{H C}= & -\frac{1}{4 \pi^{3}} \frac{C^{2}}{N} k_{p}^{2}\left(B_{H C}(0)\right. \\
& \left.+k_{p s}^{2} \int_{0}^{\infty} r B_{H C}(r) \exp \left[i k_{p s} r\right] d r\right) \\
q^{G G=} & \frac{1}{15 \pi^{3}} \frac{G H^{2}}{N k_{p}^{2}}\left(\left[4 C^{2} G+4 N H G+N H^{2}\right] B_{G G}(0)\right. \\
& \left.+4 C^{2} G k_{p s}^{2} \int_{0}^{\infty} r B_{G G}(r) \exp \left[i k_{p s} r\right] d r\right) \\
q^{C C}= & +\frac{1}{8 \pi^{3}} \frac{C^{2}}{N} k_{p}^{2}\left(B_{C C}(0)\right. \\
q^{G C}= & \frac{1}{3 \pi^{3}} \frac{\alpha^{2} M G}{P_{d}} k_{p}^{2}\left(B_{G C}(0)\right. \\
& \left.+k_{p s}^{2} \int_{0}^{\infty} r B_{G C}(r) \exp \left[i k_{p s} r\right] d r\right)
\end{aligned}
$$

Here, $B_{H H}, B_{H C}, B_{H G}, B_{G G}, B_{G C}, B_{C C}$ denote the (cross-) correlation functions of the random fields $\widetilde{H}, \widetilde{G}$, and $\widetilde{C}$ defined by Eq. (19). In the derivation of the $Q_{i k}^{(1)}$ terms we assumed that the random media realizations are statistically isotropic and therefore the correlation functions depend only on $r$. The upper bar denoting the background properties is omitted. It is important to note that Eqs. (43) -(48) will provide a correction term to the background $P$ wave number [see Eq. (36)], which exclusively accounts for conversion scattering into Biot's slow wave. The separation of terms in the operator $Q_{i k}^{(1)}$ that describe the scattering process from fast into Biot's slow $P$ wave and all other scattering processes is possible because of the low-frequency assumption [Eq. (3)]. In other words, because of the large separation between the two characteristic frequencies for ordinary elastic scattering and conversion scattering into Biot's slow wave, we can distinguish between the different scattering processes, using the frequency dependency of the corresponding terms in the scattering equation (see also Appendix B).

We will now assume that all correlation functions are of the same functional form and only differ by theirs variances, i.e., $B_{X Y}=\sigma_{X Y}^{2} B(r)$ with $B(0)=1$ and $B(\infty)=0$. Substituting then expressions (43)-(48) into Eq. (36) we obtain the final result for the effective $P$ wave number,

$$
\bar{k}_{p}=k_{p}\left(1+\Delta_{2}+\Delta_{1} k_{p s}^{2} \int_{0}^{\infty} r B(r) \exp \left[i k_{p s} r\right] d r\right),
$$

with the dimensionless coefficients

$$
\begin{aligned}
\Delta_{1}= & \frac{\alpha^{2} M}{2 P_{d}}\left(\sigma_{H H}^{2}-2 \sigma_{H C}^{2}+\sigma_{C C}^{2}+\frac{32}{15} \frac{G^{2}}{H^{2}} \sigma_{G G}^{2}\right. \\
& \left.-\frac{8}{3} \frac{G^{2}}{H} \sigma_{H G}^{2}+\frac{8}{3} \frac{G}{H} \sigma_{G C}^{2}\right), \\
= & \frac{\alpha^{2} M}{2 P_{d}}\left(\left\langle\left(\varepsilon_{H}-\frac{4}{3} \frac{G}{H} \varepsilon_{G}-\varepsilon_{C}\right)^{2}\right)+\frac{16}{45} \frac{G^{2}}{H^{2}} \sigma_{G G}^{2}\right), \\
\Delta_{2}= & \Delta_{1}+\frac{1}{2} \sigma_{H H}^{2}-\frac{4}{3} \frac{G}{H} \sigma_{H G}^{2}+\left(\frac{4 G}{H}+1\right) \frac{4}{15} \frac{G}{H} \sigma_{G G}^{2}
\end{aligned}
$$

The structure of the effective $P$ wave number can be explained as follows: Due to the presence of random inhomogeneities, there are two terms added to the background wave number $k_{p}$. The first term, $\Delta_{2}$, is frequency independent and consists in a weighted sum of the variances of the random fields $\widetilde{H}, \widetilde{G}$, and $\widetilde{C}$. The second term is frequency dependent and contains an integral over the correlation function multiplied by a weighted sum of the variances, $\Delta_{1}$. It is important to note that the expression for $\bar{k}_{P}$ describes only the process of conversion scattering from fast into slow $P$ waves. The contribution of purely elastic scattering is left out. The corresponding result would include additional terms involving the correlation functions $B_{H H}, B_{G G}$, and $B_{H G}$ that describe the elastic scattering ( $P$ to $P$ and $S$ waves) and produces the typical Rayleigh frequency dependence for attenuation. ${ }^{23}$ In other words, the second and third terms of the effective $P$ wave number (49) correspond to the mechanism of waveinduced fluid flow only. Therefore, an analysis of the properties of $\bar{k}_{p}$ gives insight into the relationship between the properties of elastic waves and wave-induced flow. By definition, the real part of $\bar{k}_{p}$ is related to the phase velocity $v$ through $v(\omega)=\omega / \Re\left\{\bar{k}_{p}\right\}$, whereas the imaginary part yields the attenuation coefficient $\gamma: \gamma(\omega)=\mathfrak{T}\left\{\bar{k}_{p}\right\}$. From the structure of Eq. (49), it can be seen that the phase velocity of the coherent wave in an equivalent medium is smaller than in the background medium. By inspection we find that $\gamma>0$, that is, the coherent wave is exponentially damped. A detailed analysis of attenuation and velocity dispersion in an equivalent medium is presented in a companion paper.

\section{RANGE OF APPLICABILITY}

The derivation of the dispersion relation (49) and the associated results on attenuation and phase velocity dispersion are based on several assumptions that restrict their range of applicability. The main restriction on our results is due to the use of the first-order statistical smoothing approximation. In the acoustic case the applicability condition of this approximation can be written as ${ }^{16}$

$$
\sigma_{n}^{2}(k a)^{2} \ll 1
$$

where $\sigma_{n}^{2}$ denotes the variance of the velocity fluctuations, $k$ is the wave number of a wave propagating in the homogeneous background, and $a$ is a characteristic length scale associated with the size of the inhomogeneities. Condition (53) ensures that the correction terms to the background wave 
number [similar to those in Eq. (49)] are small. More precisely, this condition was obtained by analyzing the next term in the equation for the kernel-of-mass operator $Q$ and requiring that the difference between the correction terms from the first-order statistical smoothing and that of the next, higher-order approximation is small. It is natural to assume that a similar condition must hold in the poroelastic case.

Using the first-order statistical smoothing the kernel-ofmass operator $\mathbf{Q}$ can be approximated by $\mathbf{Q}^{B} \approx\left\langle\widetilde{\mathbf{L}} \mathbf{G}^{0} \widetilde{\mathbf{L}}\right\rangle[\mathrm{Eq}$. (29)]. The next term in the infinite series for $\mathbf{Q}$ reads as

$$
\mathbf{Q}^{(2)}=\langle\widetilde{\mathbf{L}} \overline{\mathbf{G}} \widetilde{\mathbf{L}}\rangle,
$$

where $\overline{\mathbf{G}}$ is the matrix of the mean Green's tensors found from the first-order statistical smoothing. To compute $\mathbf{Q}^{(2)}$, we assume that there is no multiple scatter of slow $P$ waves into slow $P$ waves and, therefore, the mean Green's tensor component $\bar{g}_{33}^{F}$ is still of the form (35). Then we can derive from Eq. (33) an equation for the effective $P$ wave number similar to (36), but now involving $q_{33}^{(1)}$ determined from (54). The computation of $q_{33}^{(1)}$ according to (54) is analogous to that shown in the previous section and results in an effective $P$ wave number $\bar{k}_{p 2}$ :

$$
\bar{k}_{p 2} \approx k_{p}+\Delta_{2} k_{p}+\Delta_{1} \bar{k}_{p} k_{p s}^{2} \int_{0}^{\infty} r B(r) \exp \left[i k_{p s} r\right] d r,
$$

with $\bar{k}_{p}$ given by (49). Let us compare this result with the result for the effective wave number using first-order statistical smoothing [Eq. (49)]. Assuming that $\bar{k}_{p}$ can be represented as a sum of background wave number $k_{p}$ and a correction term $\Delta k$, the difference between (55) and (49) becomes

$$
\bar{k}_{p 2}-\bar{k}_{p}=\Delta k \Delta_{1} k_{p s}^{2} \int_{0}^{\infty} r B(r) \exp \left[i k_{p s} r\right] d r .
$$

A necessary condition for the significance of the correction term using the first-order statistical smoothing $\Delta k$ is the smallness of the difference (56) compared to $\Delta k$ :

$$
\left|\frac{\bar{k}_{p 2}-\bar{k}_{p}}{\Delta k}\right| \ll 1 .
$$

Using for instance the correlation function $B(r)$ $=\exp (-|r| / a)$, we obtain, from (56) and (57),

$$
\max \left\{\Delta_{1}\left(\left|k_{p s}\right| a\right)^{2}, \Delta_{2}\right\} \ll 1 .
$$

Relation (58) gives an estimate of the applicability of the first-order statistical smoothing approximation. That is, our results can be used in the case of weak-contrast media and weak wavefield fluctuations.

Physically, the existence of slow $P$ waves is associated with the equilibration of pore pressure that at low frequencies is controlled by the diffusion equation with diffusion length ${ }^{18} \lambda_{D}=\sqrt{\kappa_{0} N / \omega \eta}$. Therefore, the interplay between $\lambda_{D}$ and the correlation length $a$ defines two different regimes. If $\lambda_{D}>a$ then the wave-induced pressure disturbance is equilibrated. This relation holds for low frequencies and thus defines the low-frequency or relaxed regime. Conversely, if $\lambda_{D}<a$ then there is not enough time for the pore pressure to relax. This unrelaxed case occurs at high frequencies. A characteristic frequency that separates these low- and high-frequency regimes can be written as

$$
\omega_{c}=\frac{\kappa_{0} N}{a^{2} \eta} .
$$

Note that this characteristic frequency is identical to the frequency where maximal attenuation occurs. ${ }^{24}$ The existence of both regimes within our wavefield approximation, which is based on the low-frequency approximation of Biot's equations, is only possible if

$$
\omega_{c} \ll \omega_{\mathrm{B}},
$$

which imposes the additional condition for the average size of the mesoscopic inhomogeneities,

$$
a^{2} \gg \frac{\kappa_{0} N}{\eta \omega_{\mathrm{B}}} .
$$

Conditions (58) and (61) define the range of applicability of our results. Whereas condition (60) is a necessary condition for the validity of our results, there exists another condition for the observability of wave-induced flow. Only if $\omega_{c}$ is sufficiently different from the characteristic frequency, where elastic scattering of the fast wave modes dominates (i.e., $P$ $\rightarrow P$ and $P \rightarrow S$ ), it is possible to distinguish between the two processes. Elastic scattering will dominate at frequency $\omega_{S}$ $=c / a$, where $c$ is the phase velocity of the fast $P$-wave mode. Therefore, the observability condition $\omega_{S} \gtrless \omega_{c}$ imposes $a^{2} \gtrless \kappa_{0}^{2} N \rho /\left(\eta^{2} H\right)$.

\section{REDUCTION TO THE 1-D CASE}

In order to further substantiate our results, we analyze their connection with the known 1-D result. For a system of randomly layered porous media an effective, complex $P$ wave number was obtained earlier by Gurevich and Lopatnikov; ${ }^{17}$ see their equation (56). This 1-D result is also based on the method of (first-order) statistical smoothing. If only the parameter $M$ fluctuates, the 1-D result can be rewritten as

$$
\bar{k}_{p}^{1 \mathrm{D}}=k_{p}^{*}\left[1+\frac{1}{2} \mathrm{i} \frac{\alpha^{2} M}{P_{d}} k_{p s}^{*} \int_{0}^{\infty} d z B_{M M}(z) \exp \left[\mathrm{i} k_{p s}^{*} z\right]\right],
$$

where $k_{p}^{*}$ and $k_{p s}^{*}$ denote effective wave numbers that involve effective parameters $H^{*}, N^{*}$, and effective densities $\rho^{*}, q^{*}$. These effective parameters can be computed according to the so-called poroelastic Backus averaging. ${ }^{17,18,28} \mathrm{By}$ neglecting terms higher than $O\left(\epsilon^{2}\right)$-which is the overall precision of the weak-fluctuation approximation-we can replace $k_{p s}^{*}$ with $k_{p s}$. The effective parameter $H^{*}$ is computed according to

$H^{*}=P_{d}+\alpha^{2} M^{*}=P_{d}+\alpha^{2}\left\langle\frac{1}{M}\right\rangle^{-1} \approx H\left[1-\frac{\alpha^{2} M}{H} B_{M M}(0)\right]$.

Then the effective $P$ wave number $k_{p}^{*}$ can be expressed as 


$$
k_{p}^{*}=\omega \sqrt{\frac{\rho^{*}}{H^{*}}} \approx k_{p}\left[1+\frac{1}{2} \frac{\alpha^{2} M}{H} B_{M M}(0)\right] .
$$

Using (64) Eq. (62) can be written in the form

$$
\begin{aligned}
\bar{k}_{p}^{1 \mathrm{D}}= & k_{p}\left[1+\frac{\alpha^{2} M}{2 H} B_{M M}(0)\right. \\
& \left.+\frac{1}{2} \mathrm{i} \frac{\alpha^{2} M}{P_{d}} k_{p s} \int_{0}^{\infty} d z B_{M M}(z) \exp \left[\mathrm{i} k_{p s} z\right]\right] .
\end{aligned}
$$

We will now show that the 1-D result (65) can be recovered from the 3-D result. To do so, we analyze the expression for the $Q_{i k}$ tensor component in the wave number domain $q_{33}$ that is of the form [cf. Eq. (B5)]

$$
q_{33} \propto \int d^{3} \mathbf{r} B(\mathbf{r}) \frac{\exp \left[\mathrm{i} k_{p s} R\right]}{R} \exp [-\mathrm{i} \mathbf{K} \cdot \mathbf{r}],
$$

where we used only the poroelastic part of $G_{i j, i j}^{F}$ and $R$ $=|\mathbf{r}|$. Note that in order to obtain Eq. (66) it is not necessary to assume statistical isotropy. We are now considering the following limiting situation.

(a) To degenerate the 3-D random medium into a 1-D random medium we stretch the correlation lengths perpendicular to the direction of wave propagation, $a_{\perp}$, to infinity so that the correlation function becomes only a function of $z$ with parameter $a_{\|}$, i.e., the correlation length parallel to the direction of wave propagation. Obviously, if the wave propagates mainly in the $z$ direction we can also write the spatial wave vector as $\mathbf{K} \approx\left(0,0, k_{p}\right)^{T}$.

(b) Since in such a 1-D random medium there are only two directions of wave propagation $( \pm z)$, we can use the small-angle approximation ${ }^{16}$ (or Fresnel approximation) of the propagator-like term $\exp \left[\mathrm{i} k_{p s} R\right] / R$ :

$$
\frac{\exp \left[\mathrm{i} k_{p s} R\right]}{R} \approx \frac{\exp \left[\mathrm{i} k_{p s} z\right]}{z} \exp \left[\frac{\mathrm{i} k_{p s} r_{t}^{2}}{2 z}\right],
$$

where $r_{t}$ denotes the absolute value of the transverse coordinate vector $\mathbf{r}_{t}=(x, y)^{T}$. Introducing the simplifications proposed in (a) and (b) into Eq. (66) and using cylindrical coordinates $d^{3} \mathbf{r}=d z d r_{t} d \phi r_{t}$, we obtain

$$
\begin{aligned}
q_{33} \propto 4 & \pi \int_{0}^{\infty} d z B(z) \frac{\exp \left[\mathrm{i} k_{p s} z\right]}{z} \exp \left[-\mathrm{i} k_{p} z\right] \\
& \times \int_{0}^{\infty} d r_{t} r_{t} \exp \left[\frac{\mathrm{i} k_{p s} r_{t}^{2}}{2 z}\right] .
\end{aligned}
$$

The low-frequency condition $k_{p} / k_{p s} \ll 1$ means that we can replace the exponential $\exp \left[-\mathrm{i} k_{p} z\right]$ by 1 . The integral with respect to $r$ always converges because $k_{p s}$ is complex and produces an exponential decreasing multiplier. After performing the integration and substituting the result into Eq. (36), we obtain

$$
\begin{aligned}
\bar{k}_{p}= & k_{p}\left[1+\frac{\alpha^{2} M}{2 H} B_{M M}(0)\right. \\
& \left.+\frac{1}{2} \mathrm{i} \frac{\alpha^{2} M}{P_{d}} k_{p s} \int_{0}^{\infty} d z B_{M M}(z) \exp \left[\mathrm{i} k_{p s} z\right]\right],
\end{aligned}
$$

which is identical to Eq. (65).

In conclusion, the 1-D effective $P$ wave number can be exactly obtained from the 3-D result. We note that we have only considered the particular case of $M$ fluctuations. However, the approach can be also applied in the case of other parametrizations.

\section{CONCLUSIONS}

In the framework of the theory of wave propagation in random media we analyzed the properties of the coherent wave propagating in poroelastic random media. Neglecting the ordinary elastic scattering, we only accounted for conversion scattering from fast $P$ into Biot's slow $P$ wave. This process of conversion scattering is equivalent to the mechanism of pore-pressure relaxation due to wave-induced perturbations. Thus, our results describe the relationship between the dynamic properties of the coherent wavefield and the mechanism of wave-induced fluid flow. In particular, we have derived an explicit expression for the effective $P$ wave number [Eq. (49)] by applying first-order statistical smoothing of Biot's equations of poroelasticity with randomly varying coefficients. This wave number is complex and involves an integral over the correlation properties of the medium fluctuations. From this result it can be seen that the associated phase velocity is smaller than in the homogeneous background medium and that the wave is exponentially damped. We have shown that the previously reported effective $P$ wave number for randomly layered media ${ }^{17}$ can be derived from the more general 3-D result.

Our approach is limited to the case of weak-contrast, mesoscopic inhomogeneities [conditions (58) and (61)]. In this paper we focused the analysis to wave propagation in statistically isotropic random media. However, the results can be probably generalized to the case of statistically anisotropic random media. An advantage of the statistical approach is its flexibility to handle complex geometrical distributions of the inhomogeneities. Only the spatial correlation of the fluctuations need to be known in order to compute the dynamic wavefield attributes. A detailed analysis of frequency dependencies of attenuation and dispersion due to wave-induced fluid flow and the potential applicability of the results to real rocks will be the subject of a companion paper. $^{24}$

\section{ACKNOWLEDGMENTS}

This work was kindly supported by the Deutsche Forschungsgemeinschaft (Contract MU 1725/1-1), CSIRO Petroleum, and the Center of Excellence for Exploration and Production Geophysics.

\section{APPENDIX A: POROELASTIC GREEN'S TENSORS}

The complete set of Green's tensors for a homogeneous and isotropic poroelastic continuum-including electroseismic coupling - was derived by Pride and Haartsen. ${ }^{25}$ We reproduce only those parts of the Green's tensors, which are related to poroelastic wave propagation. Furthermore, we can simplify these tensors for low frequencies with $\left|k_{p}\right| /\left|k_{p s}\right|$ $\ll 1$. We obtain 


$$
\begin{aligned}
G_{i j}^{F}\left(\mathbf{r}-\mathbf{r}_{0}\right)= & \frac{1}{4 \pi \rho \omega^{2}}\left(\left[k_{s}^{2} \delta_{i j}+\partial_{i} \partial_{j}\right] \frac{e^{\mathrm{i} k_{s} R}}{R}-\partial_{i} \partial_{j} \frac{e^{\mathrm{i} k_{p} R}}{R}\right) \\
& -\frac{C^{2}}{H^{2}} \frac{1}{4 \pi q \omega^{2}} \partial_{i} \partial_{j} \frac{e^{\mathrm{i} k_{p s} R}}{R} \\
G_{i j}^{f}\left(\mathbf{r}-\mathbf{r}_{0}\right)= & \frac{C}{H} \frac{1}{4 \pi q \omega^{2}} \partial_{i} \partial_{j} \frac{e^{\mathrm{i} k_{p s} R}}{R} \\
G_{i j}^{w}\left(\mathbf{r}-\mathbf{r}_{0}\right)= & -\frac{1}{4 \pi q \omega^{2}} \partial_{i} \partial_{j} \frac{e^{\mathrm{i} k_{p s} R}}{R},
\end{aligned}
$$

where $R=\left|\mathbf{r}-\mathbf{r}_{0}\right|$. In homogeneous and isotropic media the Green's tensors only depend on $R$. In the low-frequency version of Biot's equations, the wave numbers of fast $P, S$, and slow $P$ waves are defined as

$$
k_{p}=\omega \sqrt{\frac{\rho}{H}}, \quad k_{s}=\omega \sqrt{\frac{\rho}{G}}, \quad k_{p s}=\sqrt{\frac{i \omega \eta}{\kappa_{0} N}}=\omega \sqrt{\frac{q}{N}},
$$

where $N=M P_{d} / H$. Note that the first three terms of $G_{i j}^{F}$ are formally identical to the elastodynamic Green's tensor. ${ }^{29}$ Indeed, in the elastic limit $\left(K_{d} \rightarrow K_{g}, \alpha \rightarrow 0\right.$ and $\phi \rightarrow 0, \kappa_{0}$ $\rightarrow 0$ ), the set of Green's tensors (A1)-(A3) reduces to the single elastodynamic Green's tensor,

$G_{i j}^{\text {elast }}\left(\mathbf{r}-\mathbf{r}_{0}\right)=\frac{1}{4 \pi \rho \omega^{2}}\left(\left[k_{s}^{2} \delta_{i j}+\partial_{i} \partial_{j}\right] \frac{e^{\mathrm{i} k_{s} R}}{R}-\partial_{i} \partial_{j} \frac{e^{\mathrm{i} k_{p} R}}{R}\right)$,

where the $P$ wave number is now given by $k_{p}$ $=\omega \sqrt{\rho /\left(K_{d}+4 / 3 G\right)}$.

We define the spatial Fourier transform pair in the following way:

$G_{i j}\left(\mathbf{r}-\mathbf{r}^{\prime}\right)=\int d^{3} \mathbf{K} g_{i j}(\mathbf{K}) \exp \left[i \mathbf{K} \cdot\left(\mathbf{r}-\mathbf{r}^{\prime}\right)\right]$,

$g_{i j}(\mathbf{K})=\frac{1}{(2 \pi)^{3}} \int d^{3}\left(\mathbf{r}-\mathbf{r}^{\prime}\right) G_{i j}\left(\mathbf{r}-\mathbf{r}^{\prime}\right) \exp \left[-i \mathbf{K} \cdot\left(\mathbf{r}-\mathbf{r}^{\prime}\right)\right]$.

In the wave number domain the Green's tensors (A1)-(A3) read as

$$
\begin{aligned}
g_{i j}^{F}(\mathbf{K})= & -\frac{1}{8 \pi^{3}} \frac{1}{\rho \omega^{2}}\left(\frac{k_{s}^{2} \delta_{i j}-K_{i} K_{j}}{k_{s}^{2}-K^{2}}+\frac{K_{i} K_{j}}{k_{p}^{2}-K^{2}}\right) \\
& -\frac{1}{8 \pi^{3}} \frac{C^{2}}{H^{2}} \frac{1}{q \omega^{2}} \frac{K_{i} K_{j}}{k_{p s}^{2}-K^{2}}, \\
g_{i j}^{f}(\mathbf{K})= & \frac{1}{8 \pi^{3}} \frac{C}{H} \frac{1}{q \omega^{2}} \frac{K_{i} K_{j}}{k_{p s}^{2}-K^{2}}, \\
g_{i j}^{w}(\mathbf{K})= & -\frac{1}{8 \pi^{3}} \frac{1}{q \omega^{2}} \frac{K_{i} K_{j}}{k_{p s}^{2}-K^{2}} .
\end{aligned}
$$

\section{APPENDIX B: COMPUTATION OF THE OPERATOR $Q_{i k}^{(1)}$}

In this appendix we compute the kernel-of-mass operator $Q_{i k}^{(1)}\left(\mathbf{r}^{\prime}-\mathbf{r}^{\prime \prime}\right)$ defined in Eq. (37). First we note that the three terms in Eq. (37) are of the same structure,

$$
Q_{i k}\left(\mathbf{r}^{\prime}-\mathbf{r}^{\prime \prime}\right)=\left\langle\left(L_{i j}^{(X)}\right)^{\prime} \quad G_{j l}^{Z}\left(\mathbf{r}^{\prime}-\mathbf{r}^{\prime \prime}\right) \quad\left(L_{l k}^{(Y)}\right)^{\prime \prime}\right\rangle,
$$

where $X=1,2, Y=1,2, Z=F, f, w$ while single and double primes indicate whether in the differential operator $L_{i j}$ differentiation is with respect to $\mathbf{r}^{\prime}$ or $\mathbf{r}^{\prime \prime}$. It is therefore sufficient to present the detailed manipulations only for one term. For example, for the differential operators $\left(L_{i j}^{(1)}\right)^{\prime}=\partial_{i}^{\prime} \widetilde{H}^{\prime} \partial_{j}^{\prime}$ and $\left(L_{i j}^{(2)}\right)^{\prime \prime}=\partial_{l}^{\prime \prime} \widetilde{C}^{\prime \prime} \partial_{k}^{\prime \prime}$, we have

$$
Q_{i k}=\left\langle\partial_{i}^{\prime} \widetilde{H}^{\prime} \partial_{j}^{\prime} \quad G_{j l}^{Z}\left(\mathbf{r}^{\prime}-\mathbf{r}^{\prime \prime}\right) \quad \partial_{l}^{\prime \prime} \widetilde{C}^{\prime \prime} \partial_{k}^{\prime \prime}\right\rangle .
$$

We now use partial integrations to shift the spatial derivatives $\partial_{j}^{\prime}$ and $\partial_{l}^{\prime \prime}$ to the Green's function [and make use of the property $G^{Z}(R \rightarrow \infty)=0$ ]. Averaging Eq. (B2) yields

$$
Q_{i k}=\partial_{i}^{\prime} \quad\left(B_{H C}\left(\mathbf{r}^{\prime}-\mathbf{r}^{\prime \prime}\right) \quad G_{j l, j l}^{Z}\left(\mathbf{r}^{\prime}-\mathbf{r}^{\prime \prime}\right)\right) \quad \partial_{k}^{\prime \prime},
$$

where we made use of the assumption of statistically homogeneous random fields [Eq. (19)]. The Fourier transform of $Q_{i k}$ is defined as

$$
\begin{aligned}
q_{i k}(\mathbf{K})= & \frac{1}{8 \pi^{3}} \int d^{3}\left(\mathbf{r}^{\prime}-\mathbf{r}^{\prime \prime}\right) Q_{i k}\left(\mathbf{r}^{\prime}-\mathbf{r}^{\prime \prime}\right) \exp \left[-\mathrm{i} \mathbf{K} \cdot\left(\mathbf{r}^{\prime}\right.\right. \\
& \left.\left.-\mathbf{r}^{\prime \prime}\right)\right] .
\end{aligned}
$$

Noting that spatial derivatives correspond to multiplications in the wave number domain $\left(\partial_{j} \leftrightarrow \mathrm{i} K_{j}\right)$, we can express $q_{i k}$ as

$$
q_{i k}(\mathbf{K})=\frac{-K_{i} K_{k}}{8 \pi^{3}} \int d^{3} \mathbf{r} B_{H C}(\mathbf{r}) G_{j l, j l}^{Z}(\mathbf{r}) \exp [-\mathrm{i} \mathbf{K} \cdot \mathbf{r}] .
$$

Next, we need to compute the spatial derivatives of the Green's tensors. The poroelastic part of Green's tensor in Eq. (A1) as well as Green's tensors in Eqs. (A2) and (A3) are of the form $G_{i j}^{\text {poro }} \propto \partial_{i} \partial_{j}\left(e^{\mathrm{i} k_{p s} R} / R\right)$. After differentiation we obtain

$$
G_{i j, i j}^{\mathrm{poro}}(\mathbf{r}) \propto-k_{p s}^{2}\left(k_{p s}^{2} \frac{e^{\mathrm{i} k_{p s} R}}{R}+4 \pi \delta(\mathbf{r})\right) .
$$

Analogously, for the derivatives of the elastic part of Green's tensor (A1) given by equation (A5) we find

$$
G_{i j, i j}^{\text {elast }}(\mathbf{r}) \propto-k_{p}^{2}\left(k_{p}^{2} \frac{e^{\mathrm{i} k_{p} R}}{R}+4 \pi \delta(\mathbf{r})\right) .
$$

Note that the derivatives in (B6) and (B7) are of the same functional form, despite the fact that $G_{i j}^{\text {elast }}$ includes $S$ wave contributions. These contributions, however, cancel out when $G_{i j, i j}^{\text {elast }}$ is computed. The parts of the differential operator $L_{i k}^{(1)}$ involving fluctuations of the shear modulus require the computation of the terms $G_{33,33}^{\text {elast }}$ and $G_{3 j, 3 j}^{\text {elast }}$. It can be shown that these derivatives can be expressed in terms of $G_{i j, i j}$ :

$$
\begin{aligned}
G_{33,33} & =G_{i j, i j}\left(\frac{1}{2} \int_{0}^{\pi} d \theta \sin \theta \cos ^{4} \theta+O\left(k_{p}^{2} R^{2}\right)\right) . \\
& =G_{i j, i j}\left(\frac{1}{5}+O\left(k_{p}^{2} R^{2}\right)\right) .
\end{aligned}
$$

Analogously,

$$
G_{3 j, 3 j}=G_{i j, i j}\left(\frac{1}{3}+O\left(k_{p}^{2} R^{2}\right)\right) .
$$


Neglecting terms of the order $O\left(k_{p}^{2} R^{2}\right)$ will produce no additional inaccuracy because of the restriction to low frequencies. Because of the same argument, we also neglect the first term in (B7). It is this term that is responsible for dynamic effects of elastic scattering and that is left out in the present analysis.

Inserting expressions (B6) and (B7) into Eq. (B5), introducing spherical coordinates, assuming statistical isotropy $[B(\mathbf{r})=B(|\mathbf{r}|)]$, and integrating over the angular coordinates, we obtain

$$
\begin{aligned}
q_{33}= & -\frac{1 C^{2}}{4 \pi^{3} N}\left(k_{p}^{2} B_{H C}(0)\right. \\
& \left.+k_{p} k_{p s}^{2} \int_{0}^{\infty} d r B_{H C}(r) \exp \left[\mathrm{i} k_{p s} r\right] \sin \left(k_{p} r\right)\right) .
\end{aligned}
$$

In accordance with our low-frequency assumption, we replace the sine function by its argument and obtain Eq. (45). Repeating these computations for the remaining terms involving correlations and cross-correlation between the random fields of $H, G$, and $C$ yields Eqs. (43)-(48).

${ }^{1}$ G. Mavko and A. Nur, “Melt squirt in aesthenosphere," J. Geophys. Res. 80, 1444-1448 (1975).

${ }^{2}$ W. F. I. Murphy, K. W. Winkler, and R. L. Kleinberg, "Acoustic relaxation, in sedimentary rocks: Dependence on grain contacts and fluid saturation," Geophysics 51, 757-766 (1986).

${ }^{3}$ T. Jones, "Pore fluids and frequency dependent wave propagation in rocks," Geophysics 51, 1939-1953 (1986).

${ }^{4}$ J. E. White, Underground Sound: Application of Seismic Waves (Elsevier, Amsterdam, 1983).

${ }^{5}$ W. F. Murphy, "Effects of partial water saturation on attenuation in Massilon sandstone and Vycor porous glass," J. Acoust. Soc. Am. 71, 14581468 (1982).

${ }^{6} \mathrm{G}$. A. Gist, "Interpreting laboratory velocity measurements in partially gas-saturated rocks," Geophysics 59, 1100-1109 (1994).

${ }^{7}$ R. Knight, J. Dvorkin, and A. Nur, "Acoustic signatures of partial saturation," Geophysics 63, 132-138 (1998).

${ }^{8} \mathrm{M}$. A. Biot, "Mechanics of deformation and acoustic propagation in porous media," J. Appl. Phys. 33, 1482-1498 (1962).

${ }^{9}$ J. E. White, "Computed seismic speeds and attenuation in rocks with partial gas saturation," Geophysics 40, 224-232 (1975).

${ }^{10}$ J. E. White, N. G. Mikhaylova, and F. M. Lyakhovitsky, "Low-frequency seismic waves in fluid saturated layered rocks," Izv., Acad. Sci., USSR, Phys. Solid Earth 11, 654-659 (1975).
${ }^{11}$ N. C. Dutta and H. Ode, "Attenuation and dispersion of compressional waves in fluid-filled porous rocks with partial gas saturation (White model)—Part I: Biot theory," Geophysics 44, 1777-1788 (1979).

${ }^{12}$ S. R. Pride and J. G. Berryman, "Linear dynamics of double-porosity dual-permeability materials I. governing equations and acoustic attenuation," Phys. Rev. E 68, 036603 (2003).

${ }^{13}$ S. R. Pride, J. G. Berryman, and J. M. Harris, "Seismic attenuation due to wave-induced flow," J. Geophys. Res. 109, B01201 (2004).

${ }^{14}$ D. L. Johnson, "Theory of frequency dependent acoustics in patchysaturated porous media," J. Acoust. Soc. Am. 110, 682-694 (2001).

${ }^{15}$ S. L. Lopatnikov and B. Gurevich, "Transformational mechanism of elastic wave attenuation in saturated porous media," Izv., Acad. Sci., USSR, Phys. Solid Earth 24, 151-154 (1988).

${ }^{16}$ S. M. Rytov, Y. A. Kravtsov, and V. I. Tatarskii, "Wave propagation through random media," Vol. 4 of Principles of Statistical Radiophysics (Springer-Verlag, Berlin, 1989).

${ }^{17}$ B. Gurevich and S. L. Lopatnikov, "Velocity and attenuation of elastic waves in finely layered porous rocks," Geophys. J. Int. 121, 933-947 (1995).

${ }^{18}$ A. N. Norris, "Low-frequency dispersion and attenuation in partially saturated rocks," J. Acoust. Soc. Am. 94, 359-370 (1993).

${ }^{19}$ B. Gurevich, A. P. Sadovnichaja, S. L. Lopatnikov, and S. A. Shapiro, "Scattering of a compressional wave in a poroelastic medium by an ellipsoidal inclusion," Geophys. J. Int. 133, 91-103 (1998).

${ }^{20} \mathrm{~J}$. B. Keller, "Stochastic equations and wave propagation in random media," Proc. Symp. Appl. Math. 16, 145-170 (1964).

${ }^{21}$ F. C. Karal and J. B. Keller, "Elastic, electromagnetic and other waves in random media," J. Math. Phys. 5, 537-547 (1964).

${ }^{22}$ J. J. McCoy, "On the dynamic response of disordered composites," J. Appl. Mech. 40, 511-517 (1973).

${ }^{23}$ N. Gold, S. A. Shapiro, S. Bojinski, and T. M. Müller, "An approach to upscaling for seismic waves in statistically isotropic heterogeneous elastic media," Geophysics 65, 1837-1850 (2000).

${ }^{24}$ T. M. Müller and B. Gurevich, "Wave-induced fluid flow in porous random media: Attenuation and dispersion of seismic waves," J. Acoust. Soc. Am., accepted.

${ }^{25}$ S. R. Pride and M. W. Haartsen, "Electroseismic wave properties," J. Acoust. Soc. Am. 100, 1301-1315 (1996).

${ }^{26} \mathrm{~A}$. N. Norris, "Radiation from a point source and scattering theory in a fluid-saturated porous solid," J. Acoust. Soc. Am. 77, 2012-2023 (1985).

${ }^{27}$ B. Gurevich and S. L. Lopatnikov, "Attenuation of longitudinal waves in a saturated porous medium with random inhomogeneities," Transactions (Doklady) of the USSR Academy of Sciences Earth Sci. Sections 281, 47-50 (1985).

${ }^{28}$ S. Gelinsky and S. A. Shapiro, "Poroelastic Backus-averaging for anisotropic, layered fluid and gas saturated sediments," Geophysics 62, 18671878 (1997).

${ }^{29}$ J. E. Gubernatis, E. Domany, and J. A. Krumhansl, "Formal aspects of the theory of the scattering of ultrasound by flaws in elastic materials," J. Appl. Phys. 48, 2804-2811 (1977). 